\title{
Superresolution by image scanning microscopy using pixel reassignment
}

\author{
Colin J. R. Sheppard, ${ }^{1, *}$ Shalin B. Mehta, ${ }^{2}$ and Rainer Heintzmann ${ }^{3,4,5}$ \\ ${ }^{1}$ Nanophysics, Istituto Italiano di Tecnologia, via Morego 30, 16163 Genova, Italy \\ ${ }^{2}$ Marine Biological Laboratory, Woods Hole, Massachusetts 02543, USA \\ ${ }^{3}$ Institute of Photonic Technology, Albert Einstein Str. 9, 07745 Jena, Germany \\ ${ }^{4}$ Institute of Physical Chemistry, Abbe Centre of Photonics, Friedrich-Schiller-University Jena, Helmholtzweg 5, 07743 Jena, Germany \\ ${ }^{5}$ King's College London, Randall Division of Cell and Molecular Biophysics, SE1 1UL London, UK \\ *Corresponding author: colinjrsheppard@gmail.com
}

Received June 12, 2013; accepted June 26, 2013;

posted July 8, 2013 (Doc. ID 192220); published July 30, 2013

\begin{abstract}
The effect of detector array size on resolution and signal collection efficiency of image scanning microscopy based on pixel reassignment is studied. It is shown how the method can also be employed if there is a Stokes shift in fluorescence emission wavelength. With no Stokes shift, the width of the point spread function can be sharpened by a factor of 1.53, and its peak intensity increased by a factor of 1.84. (־ 2013 Optical Society of America

OCIS codes: (170.1790) Confocal microscopy; (180.2520) Fluorescence microscopy; (180.5810) Scanning microscopy.

http://dx.doi.org/10.1364/OL.38.002889
\end{abstract}

In a recent paper, Müller and Enderlein [1] described a method for improving the spatial resolution of microscopy, based on processing the signal recorded by an imaging detector as the object is scanned relative to a focused laser spot. They call their method image scanning microscopy. Their paper was considered significant enough to be selected as an Editor's Suggestion and to be covered by a Viewpoint in the journal Physics [2]. Actually, the method is identical with one described more than 20 years ago [3]. Even more recently, other groups have explored similar methods [4,5]. In our opinion, the major strength of the method is in greatly improving the detected signal level in confocal imaging, while at the same time slightly improving upon the confocal resolution limit. This combination greatly improves upon the overall performance for practical fluorescence microscopy. The goals of this paper are to describe the extension to fluorescent dyes exhibiting a Stokes shift and to explore the effects of the size of the detector array.

In [1] the operation of the technique in terms of structured illumination is explained [6-9]. In [3], on the other hand, the method as a confocal microscope with a detector array is explained. The confocal microscope can also be thought of as a special case of structured illumination. The spinning disk microscope, where the similarity between the pinhole array and a grating structure is apparent, can be thought of in this manner as well.

Figure 1(a) shows the signal recorded from a point object at different points $v_{d}$ in the detector plane as the sample is scanned [10]. Here $v$ is the normalized transverse coordinate of the scan position, $v=(2 \pi / \lambda) n x \sin \alpha$, where $n$ is the refractive index of the immersion medium and $\alpha$ is the semi-angular aperture of the microscope objective. Similarly, $v_{d}$ is given by the analogous expression $v_{d}=(2 \pi / \lambda) x_{d} \sin \alpha_{d}$ for the detector element position. The Stokes shift is neglected for the present. Figure 1(a) shows a cross-section through the image in the direction of the detector position $v_{d}$. For $v_{d}=0$, corresponding to a point on the axis, a confocal image results. As $v_{d}$ increases, the peak of the image intensity decreases and shifts sideways, and the image is sharper, but no longer circularly symmetric. Integration of these signals over the complete plane gives rise to a scanning, nonconfocal image, equivalent to a conventional image if there is no Stokes' shift.

However, if the images from the off-axis detector points are shifted, as in Fig. 1(b), they add up to give a sharper point spread function (PSF), as shown in Fig. 1(c). To obtain this behavior, the values measured by a circular detector array of normalized radius $v_{d \text { max }}$
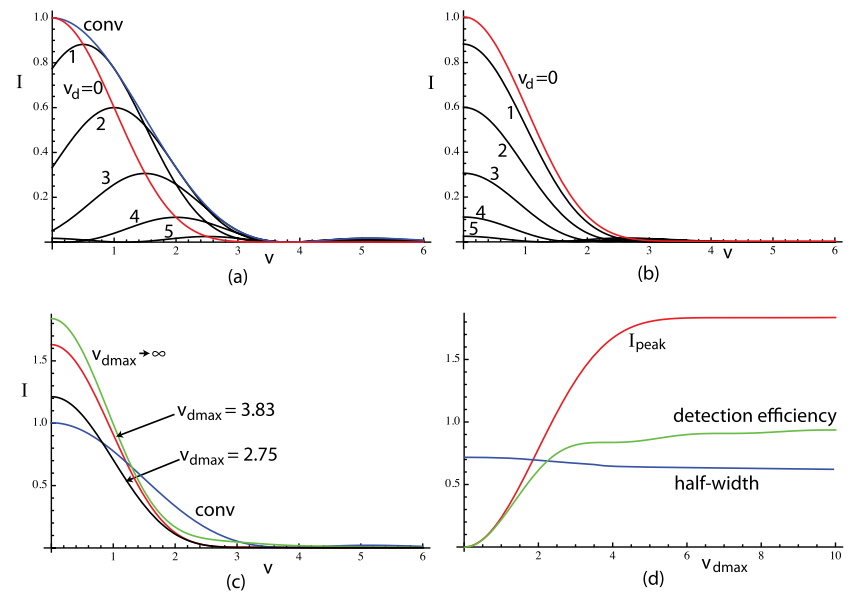

Fig. 1. (a) Cross-section through the image of a point object in a scanning microscope for a point detector displaced a normalized distance $v_{d}$ from the axis. There is assumed to be no Stokes shift. The peak intensity is reduced and shifted sideways as the offset is increased, (b) after shifting the image so that its peak is on the axis, and (c) cross-section through the (circularly symmetric) image of a point object in a scanning microscope after pixel reassignment with a detector array of normalized radius $v_{d \text { max }}$. The conventional case is also shown. (d) Peak intensity and normalized half-width of the image of a point object after pixel reassignment with a detector array of normalized radius $v_{d \text { max }}$. The half-width is normalized to unity for a conventional microscope. The detection efficiency (normalized to unity for the conventional case) is also shown. 
Table 1. Imaging Performance with Pixel Reassignment Compared with Conventional and Confocal Microscopes

$v_{d \max }$ Normalized Resolution Peak of $\eta$ Half-width Improvement PSF Factor

\begin{tabular}{llllll}
\hline Confocal & 0 & 0.720 & 1.39 & 0 & 0 \\
& 2.75 & 0.689 & 1.45 & 1.21 & 0.79 \\
& 3.83 & 0.671 & 1.49 & 1.63 & 0.84 \\
Conventional & $\infty$ & 0.652 & 1.53 & 1.84 & 1 \\
\hline
\end{tabular}

must be summed to the image point $v=v_{d} / 2$. We call this process pixel reassignment. In Fig. $\underline{1(\mathrm{c})}$ the peak intensity has been normalized to the conventional case. As $v_{d \text { max }}$ increases, the width of the PSF decreases monotonically. The intensity at the peak for different sizes of detector array is shown in Fig. 1(d), where the values have been normalized to that for a conventional microscope. This can actually be greater than for a conventional microscope, and is $4\left(1-16 / 3 \pi^{2}\right)=1.84$ for a large array.

The improvement in resolution, defined in terms of the half-width of the PSF, is given in Table 1 . For a large detector array, the improvement compared with a conventional microscope is by a factor of 1.58 . The intensity at the peak of the PSF is also shown in Table 1 . The signal recorded at a point $\mathbf{x}_{d}=x_{d}, y_{d}$ in the plane of the detector for a two-dimensional (2D) fluorescent object of strength $F\left(\mathbf{x}^{\prime}-\mathbf{x}_{s}\right)$ at scan position $\mathbf{x}_{s}$ is

$$
I\left(\mathbf{x}_{s}, \mathbf{x}_{d}\right)=\iint H_{1}\left(\mathbf{x}^{\prime}\right) F\left(\mathbf{x}^{\prime}-\mathbf{x}_{s}\right) H_{2}\left(\mathbf{x}^{\prime}-\mathbf{x}_{d}\right) \mathrm{d}^{2} \mathbf{x}^{\prime},
$$

where $H_{12}$ are the intensity PSFs for illumination and detection. Substituting $\mathbf{x}_{s}=-\mathbf{x}_{1}, \mathbf{x}_{d}=\mathbf{x}_{2}-\mathbf{x}_{1}, \mathbf{x}^{\prime}=\mathbf{x}-\mathbf{x}_{1}$, corresponding to scanning the illumination beam instead of the specimen, this can be written in the symmetrical form for the four-dimensional signal:

$$
I\left(\mathbf{x}_{1}, \mathbf{x}_{2}\right)=\iint H_{1}\left(\mathbf{x}-\mathbf{x}_{1}\right) H_{2}\left(\mathbf{x}-\mathbf{x}_{2}\right) F(\mathbf{x}) \mathrm{d}^{2} \mathbf{x} .
$$

The signal from measurement $\mathbf{x}_{d}$ is reassigned to the point $\mathbf{x}_{r}=(1-a) \mathbf{x}_{1}+a \mathbf{x}_{2}$ and summed with pinhole weighting factor $S\left(\mathbf{x}_{d}\right)$ to give

$$
\begin{aligned}
I\left(\mathbf{x}_{r}\right)= & \iiint \int H_{1}\left[\mathbf{x}-\mathbf{x}_{r}-a \mathbf{x}_{d}\right] H_{2}\left[\mathbf{x}-\mathbf{x}_{r}+(1-a) \mathbf{x}_{d}\right] \\
& \times F(\mathbf{x}) S\left(\mathbf{x}_{d}\right) \mathrm{d}^{2} \mathbf{x} \mathrm{d}^{2} \mathbf{x}_{d} .
\end{aligned}
$$

This expression is valid for any $a$, with $0 \leq a \leq 1$. The value $a=1 / 2$ corresponds to pixel reassignment as described above. If $a=1$, the system reduces to a conventional microscope, whereas if $a=0$ the system becomes a scanning (nonconfocal) microscope, i.e., equivalent to integration without reassignment.

If $S\left(\mathbf{x}_{d}\right)=1$, using a large detector, the integral in $\mathbf{x}_{d}$ in Eq. (3) gives the effective PSF as the 2D convolution of two scaled, 3D PSFs (illumination and detection PSFs). Thus, $C_{\text {eff }}(\mathbf{m})$ is the product of two scaled, illumination and detection, optical transfer functions (OTFs) in $x, y$, convolved in the axial direction. Although this was shown to be true for $a=1 / 2$ [1,3], actually this result is true for a large detector array even for the more general case when the signal is summed to the point $v=a v_{d}$, where $a$ is a constant less than unity. We find that

$$
C_{\text {eff }}(\mathbf{m})=C_{1}[(1-a) \mathbf{m}] C_{2}[a \mathbf{m}]
$$

with $C_{1}(\mathbf{m})$ and $C_{2}(\mathbf{m})$ being the excitation and emission transfer functions, respectively, at in-plane spatial frequency $\mathbf{m}$.

As $C_{1}(0)=C_{2}(0)=1$, independent of the presence of defocus, $C_{\text {eff }}(0)=1$, so that there is no optical sectioning when pixel reassignment is used with a large detector array. This can also be seen from the 3D OTF, which is given by a $1 \mathrm{D}$ convolution (in the axial direction, but a product in $x, y$ ) of two scaled OTFs. A cross-section through the resulting OTF for no Stokes shift and $a=1 / 2$ is compared with the conventional OTF in Fig. 2. The cutoff spatial frequency is doubled in all three directions, but the missing cone is still present.

The fact there is no sectioning is, of course, a major disadvantage compared with ordinary confocal microscopy, as optical sectioning is its primary attribute. But as the effective OTF is always positive for zero Stokes shift, there are fewer artifacts with defocus, which is a desirable feature. This could be an important property of the method for imaging of the projection of a thin sample, perhaps combined with filters to improve depth of focus. Optical sectioning can, however, be regained by limiting the size of the detector array, i.e., by taking $S\left(\mathbf{x}_{d}\right)=1, \mathbf{x}_{d}<\mathbf{x}_{d \text { max }} ; S\left(\mathbf{x}_{d}\right)=0, \mathbf{x}_{d}>\mathbf{x}_{d \text { max }}$. This behavior occurs because the maximum value of the intensity in the detector plane $\left|h_{1}\left(v_{d}, u\right)\right|^{2}$, where $h_{1}$ is the amplitude PSF of illumination and $u$ is the normalized defocus coordinate, for a given $v_{d}$ occurs at $u=0$ only if $v_{d}<2.747$. For larger values of $v_{d}$, the maximum tends to a point on the shadow edge $[11,12]$. This explains why the optical sectioning effect is reduced by pixel reassignment with a large detector array. Taking $v_{d \max }=2.747$, however, retains an optical sectioning effect, while giving a good compromise in resolution improvement, and detection efficiency given by $\eta=1-J_{0}^{2}\left(v_{d \max }\right)-J_{1}^{2}\left(v_{d \max }\right)[\underline{13}]$. The variation in collection efficiency with $v_{d \text { max }}$ is shown in Fig. 1(d). A value of $v_{d \max }=3.83$ corresponds to a detector array equal in size to the first dark ring of the Airy disk in the detector plane.

If there is a Stokes shift, we choose $a=1 /(1+\beta)$, where $\beta$ is the ratio of the emission wavelength $\left(\lambda_{\mathrm{em}}\right)$ to excitation wavelength $\left(\lambda_{\mathrm{ex}}\right)$, as then the cutoffs of the two OTFs are equal, thus optimizing the resolution.

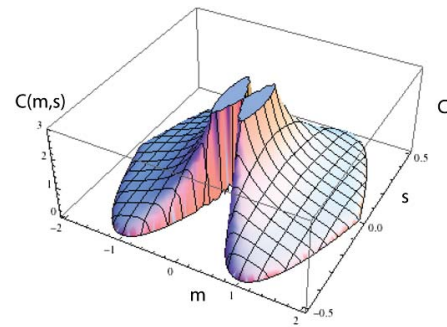

(a)

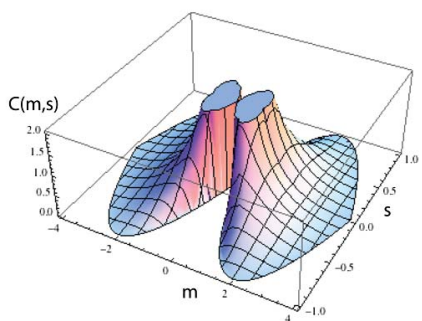

(b)
Fig. 2. 3D OTFs for (a) conventional fluorescence and (b) after pixel reassignment. There is assumed to be no Stokes shift. 

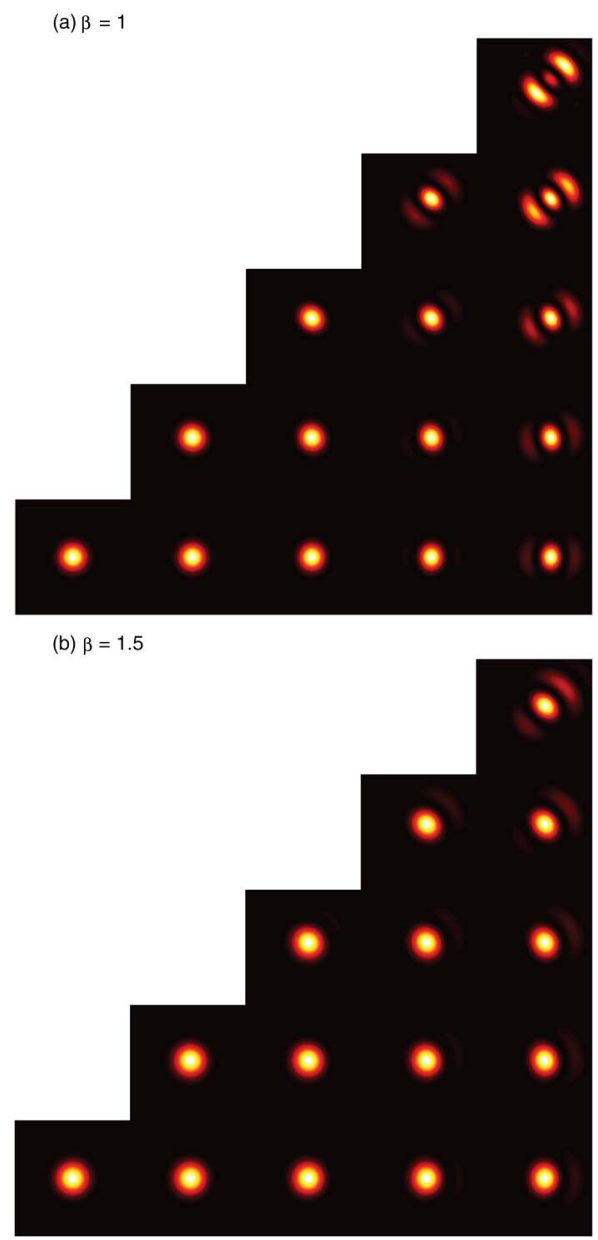

Fig. 3. Effective PSF for different pixels on a $9 \times 9$ detector array of size $9 \lambda / \mathrm{NA}$, just larger than the first zero of the Airy disc. (a) Stokes shift is neglected. (b) Stokes shift is assumed to be such that $\lambda_{\mathrm{em}} / \lambda_{\mathrm{ex}}=1.5$. The central pixel gives close to a true confocal image.

Then we find that

$$
C_{\text {eff }}(\mathbf{m})=C_{1}\left(\frac{\beta \mathbf{m}}{1+\beta}\right) C_{2}\left(\frac{\mathbf{m}}{1+\beta}\right) .
$$

The cutoff frequency is then improved by a factor $(1+\beta)$ compared with a conventional microscope, which is greater than 2 if $\beta>1$. This is also true for a confocal microscope, but the OTF for the reassignment case has greater value within the pass band. The improved cutoff frequency, in the presence of a Stokes shift, of scanning microscopy in general does not seem to be widely appreciated.

Figure 3(a) shows the effective PSF for different elements of a $9 \times 9$ element detector when Stokes shift is not present, and Fig. 3(b) shows the effective PSF for the same configuration with Stokes shift given by $\beta=1.5$. The intensity PSFs and OTFs in Figs. $\underline{3}-\underline{5}$ were computed by adapting image simulation routines from the microlith [14] package.

Media 1 (with a frame shown in Fig. 4) shows the evolution of the effective PSF as the PSFs of different detector pixels as shown in Fig. 3(b) are reassigned and summed. The reassignment leads to a $150 \%$ stronger peak

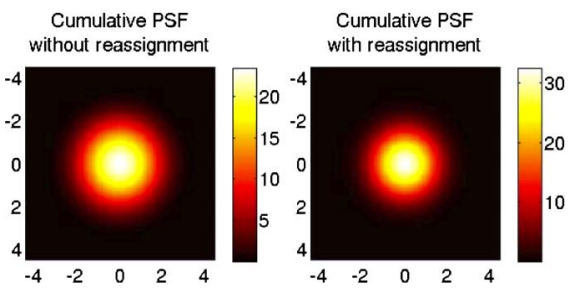

Fig. 4. Frame from Media 1: transverse PSF due to summation of effective PSFs shown in Fig. 3(b) without and with reassignment. Spatial coordinates are expressed in terms of $v=\left(2 \pi / \lambda_{\mathrm{ex}}\right) n x \sin \alpha$.

and a sharper PSF as compared to nonreassigned summation. Setting the size of the scan pixel to $1 /(2 N)$ $(N$ integer) the size of the detector pixel avoids the need for interpolation for reassignment by $v_{d} / 2$. More generally, reassignment of signal by $a v_{d}$ can be performed without interpolation by using scan pixels of size $a / N$, the size of the detector pixel.

Figure 5(a) shows the cross-section of the PSFs in the $x-z$ plane $(x, y$, transverse; $z$, axial coordinates) after summation over the array with or without reassignment, assuming no Stokes shift or a Stokes shift of $\beta=1.5$. The PSFs are compared with the PSF of the conventional microscope, which images light with wavelength $\beta \lambda$. Since Stokes shift lowers the resolution in conventional microscopy, the reassignment by $a v_{d}$ is particularly useful for regaining resolution, while also increasing signal level. Figure 5(b) shows the 2D Fourier transforms of the PSF sections shown in Fig. 5(a), i.e., the projection of the 3D OTF along the $y$-axis as per the projection-slice theorem. The projections of the OTF illustrate effectively the spatial frequency cutoffs of different schemes. We note that reassignment fills the "dips" in the OTF and provide better resolution and SNR.

It is necessary to clarify a comment about resolution in $[1,2]$. The statement "summing the intensity over the whole ... CCD" "corresponds to a conventional CLSM
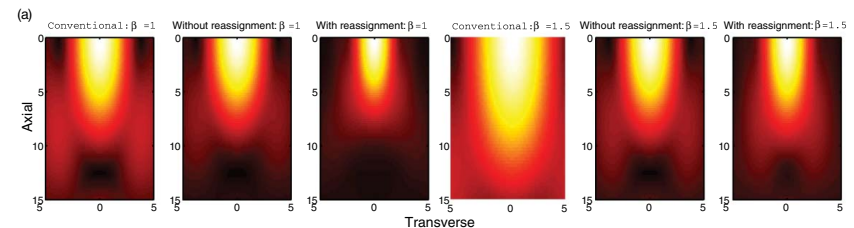

(b)
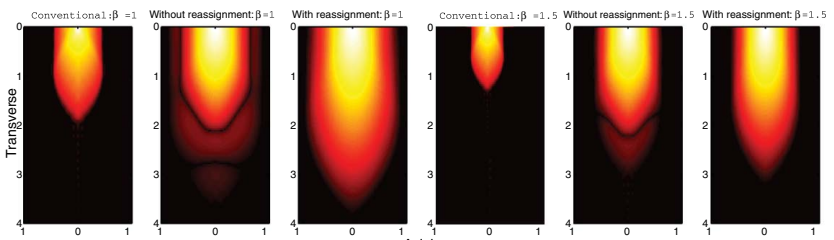

Fig. 5. (a) Comparison of the transverse and axial PSFs for a conventional microscope, and for summation over a $9 \times 9$ pixel detector array, without and with reassignment at a weighting factor of one. (b) Transverse projection of the 3D OTFs obtained by 2D Fourier transforms of PSFs shown in (a). The display gamma is 0.5 . Note that the transverse and axial axes are switched between PSF and OTF plots. Distance and spatial-frequencies are expressed in normalized coordinates of $v=\left(2 \pi / \lambda_{\mathrm{ex}}\right) n x \sin \alpha$ and $m=n x \sin \alpha / \lambda_{\mathrm{ex}}$, respectively in terms of the excitation wavelength. 
image" [1] is incorrect. It corresponds to a scanned nonconfocal image, i.e., with resolution given by the excitation wavelength. Confocal microscopy, like some other geometries of structured illumination, gives a spatial frequency cutoff twice that of a conventional microscope. (Here we assume no Stokes shift: otherwise it is improved by a factor $1+\beta$, the same as for the reassignment case, discussed above.) However the response at high spatial frequencies is very weak [15], so that the improvement in the width of the PSF is only by a factor of 1.39 . And in practice, with a nonzero pinhole size, the improvement is small [16].

This indicates the advantage of the present pixel reassignment technique, where the whole signal on the detector array is detected. Structured illumination (by fringe projection), on the other hand, has the advantages that the resolution is better than confocal microscopy because the response at high spatial frequencies is higher, and that it is a multiplex system where all points of the object are imaged simultaneously.

It is worth noting that simple pixel reassignment is by no means the only way of processing the available data. Other ways of processing include, in close analogy to subtractive imaging, weighted averaging in Fourier space and multiview deconvolution [17]. Such approaches are expected to deliver superior performance in terms of signal to noise compared to the simple reassignment approach as discussed here. Using a nonunity value of the pinhole weighting factor $S\left(\mathbf{x}_{d}\right)$ may also prove advantageous.

It is possible to implement the reassignment concept in an all-optical way, for example by expanding the beam on the scan-mirror optically by a factor of $1 / a$ before it is rescanned with (possibly the same) scan mirror on to a CCD camera [18]. As a result, the reassignment (with a weighting factor of 1 ) is automatically realized on the camera and the above considerations equally apply. This has the advantage of requiring the recording of only a single frame, with associated improved speed and reduced read noise. However, it has the potential disadvantage of not allowing for other ways of data processing, or the ability retrospectively to choose the pinhole size.

\section{References}

1. C. B. Müller and J. Enderlein, Phys. Rev. Lett. 104, 198101 (2010).

2. H. Blom and J. Widengren, Physics 3, 40 (2010).

3. C. J. R. Sheppard, Optik 80, 53 (1988).

4. A. G. York, S. H. Parekh, D. D. Nogare, R. S. Fischer, K. Temprine, M. Mione, A. B. Chitnis, C. A. Combes, and H. Shroff, Nat. Methods 9, 749 (2012).

5. G. De Luca, R. Breedijk, and E. Manders, "Point-scanning based super-resolution microscopy," presented at Focus on Microscopy, Maastricht, 2013.

6. W. Lukosz and M. Marchand, Optica Acta 10, 241 (1963).

7. M. M. A. Neil, R. Juskaitis, and T. Wilson, Opt. Lett. 22, 1905 (1997).

8. M. Gustafsson, J. Microsc. 198, 82 (2000).

9. R. Heintzmann and P. Benedetti, "Space and frequency domain reconstruction strategies in a confocal microscope based on patterned illumination and wide-field image collection," presented at Focus on Microscopy, Genoa, 2003.

10. I. J. Cox, C. J. R. Sheppard, and T. Wilson, Appl. Opt. 21, 778 (1982).

11. C. J. R. Sheppard and H. J. Matthews, J. Mod. Opt. 35, 145 (1988).

12. T. Wilson and A. R. Carlini, Appl. Opt. 27, 3791 (1988).

13. M. Born and E. Wolf, Principles of Optics, 5th ed. (Pergamon, 1975).

14. S. B. Mehta and R. Oldenbourg, "microlith: image simulation for biological phase microscopy," arXiv:1305.7149v1.

15. I. J. Cox, C. J. R. Sheppard, and T. Wilson, Optik 60, 391 (1982).

16. G. Cox and C. J. R. Sheppard, Microsc. Res. Tech. 63, 18 (2004).

17. R. Heintzmann, V. Sarafis, P. Munroe, J. Nailon, Q. S. Hanley, and T. M. Jovin, Micron 34, 293 (2003).

18. S. Roth, C. J. R. Sheppard, K. Wicker, and R. Heintzmann, "Optical photon reassignment microscopy (OPRA)," arXiv: 1306.6230. 